\title{
The Impact of the Google Cloud to Increase the Performance for a Use Case in E-Commerce Platform
}

\author{
Silvana Greca \\ Department of Informatics \\ Faculty of Natural Sciences \\ University of Tirana \\ Tirana, Albania
}

\author{
Anxhela Kosta \\ Department of Informatics \\ Faculty of Natural Sciences \\ University of Tirana \\ Tirana, Albania
}

\begin{abstract}
A massive amount of complex and huge digital data storing is caused due to the development of intelligent environments and cloud computing. Today the organizations are using Google Cloud platform to store and retrieve of all amount of their data at any time. Google offers database storage like:Cloud Datastore for NoSQL non-relational database and Cloud SQL for MySQL fully relational database. During years, enterprise organizations have accumulated growing stores of data, running analytics on that data to gain value from large information sets, and developing applications to manage data exclusively. Traditional SQL databases are used for storing and managing content of structured data, but with unstructured and complex data they have found it difficult. Based on this need, the purpose of this paper is to present a Cloud storage solution able to store huge amount of data, focused on the impact of performance and scalability for a use case on e-commerce. For this reason, we have compared MongoDB with MySQL on Google Cloud to find the best solution for our e-commerce use case.
\end{abstract}

\section{Keywords}

Cloud computing, Performance, Scalability, NoSQL Database, MongoDB, MySQL, stock, e-commerce

\section{INTRODUCTION}

The rapid development of Internet Web technology, cloud computing and distributed services, has created the need to store large amount of data in distributed databases to provide high availability and scalability. To store the information and data in a high level of security for the organizations and companies has been necessary to pass from traditional platform of storing data, from a physical host into a new platform called Google Cloud. Google Cloud is a platform that offers services for compute, storage and manage big data, security and also offers developer tools [1]. For over a decade, some organizations have been using clouds and considering it a provider. But a key challenge in porting enterprise software systems to the cloud is the migration of their database, due to the size and importance of the data it contains. Google offers database storage, which include: Cloud Datastore for NoSQL non-relational database and Cloud SQL for MySQLfully relational database.

Relational database systems have been and are efficient systems that face multiple operations for storing and managing structured data. A relational database is a collection of data items organized as a set of formally-described tables from which data can be accessed or reassembled in many different ways without having to reorganize the database tables.
Storing the unstructured and complex data in traditional SQL database is very difficult[13]. For this reason, it was necessary to create many types of database. NoSQL databases are useful for applications that deal with very large semi-structured and unstructured data. NoSQL databases do not require a default schema associated with the data. The purpose of the NoSQL databases is to increase the performance and scalability of a specific application and service.

Nowadays, e-commerce platform is very popular and is going through a transformation driven by customers who expect a seamless experience between online and in store. As a result, many retailers are turning to cloud technologies in order to meet these needs. The performance and scalability for this platform are the most important elements.

Based on the latest work [15], the purpose of this paper is to present Cloud storage solution to be able to store a huge amount of data, focused on the impact of performance and scalability for our use case. We have compared MongoDB with MySQL on Google Cloud platform, focused on the performance and scalability of specific applications and services. This comparison is done in a real implementation on Google Cloud for managing the use case that needs huge complex data storing.

This paper is organized as follows: Section 1 presents a brief overview on Google Cloud Platform, which fostered its storage platform, license type, and programming language, advantages and disadvantages. Section 2 provides a general theoretical background to e-commerce platform, their performance and scalability. Section 3 presents an implementation on Google Cloud using NoSQL database and MySQL database, on the same condition, for storing big data to an e-commerce platform. The final section (Section 4) concludes the main findings and implications of this paper.

\section{THE CHALLENGE OF PHYSICAL HOST AND CLOUD COMPUTING}

Companies use their data in many ways to meet business needs and for this reason they need a technology that must be able to process and store the on-demand business data consistently and effectively. Some of them use physical host and others dedicated host.

Dedicated hosting is an Internet hosting option in which an organization leases an entire server, which is often housed in a data center. Based on company experience the academics have identified more disadvantages for this host in report on advantages. Below are some of the cons [12]: 
- Much more expensive- The resources that are used to run and maintain a physical serverare much more expensive.

- Difficulty in managing - The physical servers are overall much harder to manage.

- Less scalable - It is almost impossible to upgrade a server without additional downtime

According to Ahmad [2] Cloud computing is basically a philosophy and design concept of computing architecture, while, it is much more complicated but yet so much simpler.

The basic concept is to separate the Applications, Operating System and Hardware from each other. Advantages of cloud computing over traditional computing include: agility, lower entry cost, device independency, location independency, and scalability [4].

To use the cloud, the user is being charged based on the subscription model. Companies can access these hosted data or applications from cloud servers over the Internet through their devices. Cloud service providers bill for each instance, like pay-as-you-go [3]. So, in Google Cloud you pay for what you use, hence offering cost transparency and better accountability. In Google Cloud the client pays for the services it uses, so it will provide better transparency in cost and accountability.

More and more businesses and research agencies [9] have identified the following key advantages for cloud:

1. Accessibility: Files in the cloud can be accessed from anywhere with an internet connection.
2. Cost savings: It will reduce the operating cost because does not depend on internal power to store information remotely.

3. Disaster recovery: All businesses should invest in an emergency backup plan and cloud is the best platform that offers this opportunity.

4. Scalability: With cloud storage, the client pays for the amount of storage he requires. If his business experiences growth, then the cloud operator can help accommodate its corresponding growth in data storage needs.

5. Speed: Tests have shown that when the cloud is supported by the right enterprise technologies, the speeds achieved can rival on site scores.

6. Storage immortality: The client can pay for the capacity and performance his business requires, and his provider can upgrade the environment to keep pace with the latest technology. This is brought on by competitive pressures from other cloud providers.

The infrastructure of cloud refers to the hardware and software components such as servers, storage, and network virtualization software. It incorporates Infrastructure as a Service (IaaS), Platform as a Service (PaaS), Software as a Service (SaaS), and provides these services like utilities, so the end users are billed by how much they used.

Figure 1 shows a hierarchical view for cloud computing [5].

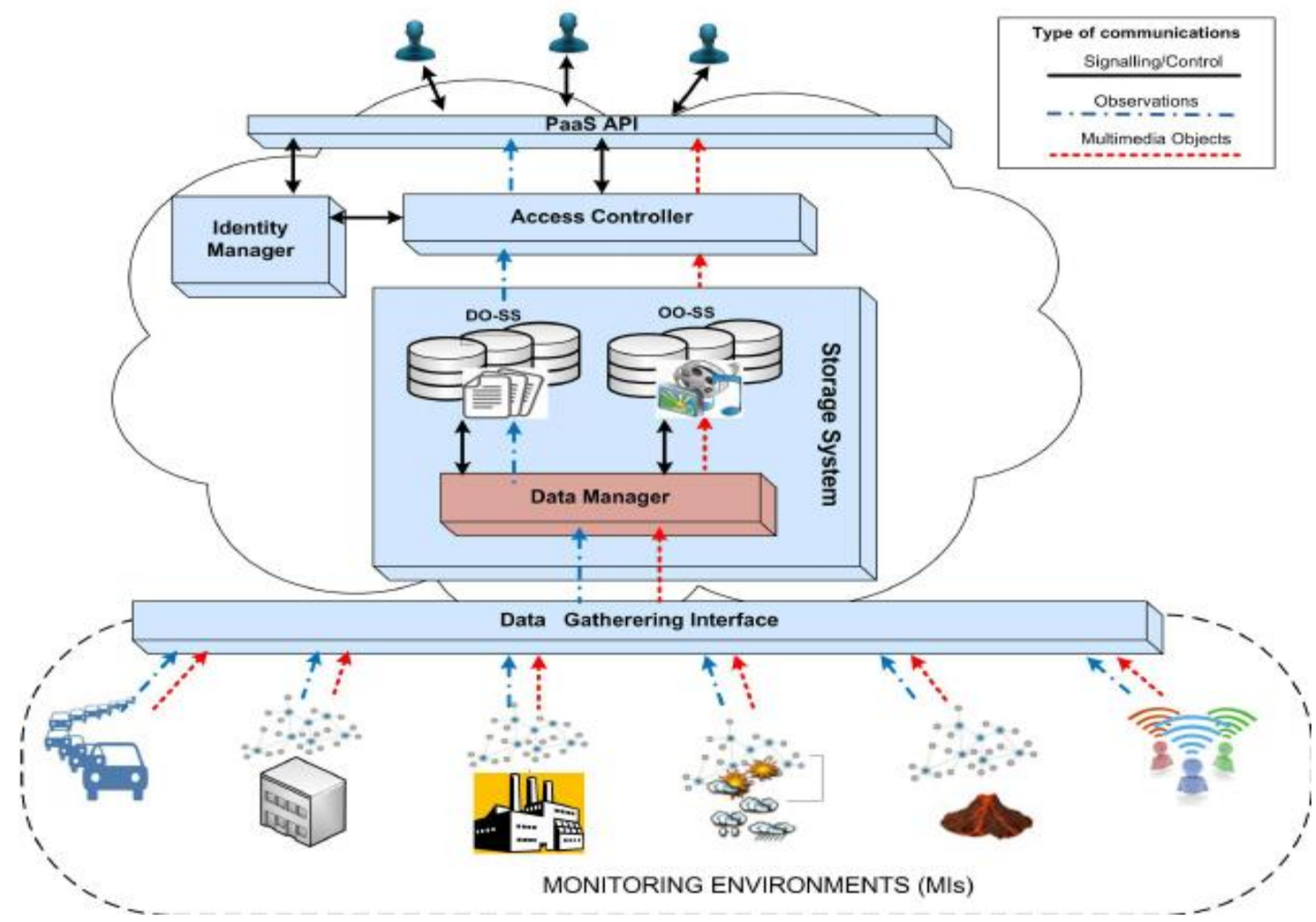

Fig 1. The Cloud storage service

The infrastructure of cloud is so popular so business and IT giants such as Google, Amazon, Microsoft and IBM have started their cloud computing infrastructure [2]. But a key challenge in porting enterprise software systems to the cloud is the migration of their databases, due to the size and importance of the data they contain. Cloud computing technology is becoming the best choice of the virtual operation process due to its features such as reliability and security. 


\section{PERFORMANCE AND SCALABILITY OF E-COMMERCE PLATFORM}

Cloud is a powerful technology to perform massive-scale and complex data, but it has necessary an environment for storing these data on cloud, a database storage. In cloud we can migrate different databases, based on the nature of the business and types of data (structured and unstructured). Let describe shortly what structure, semi-structured and unstructured data are [6]:

- $\quad$ Structured data are often managed SQL, a programming language created for managing and querying data in RDBMS. Structured data are easy to input, query, store, and analyze [13].

- Semi-structured data are data that do not follow a conventional database system. Semi-structured data may be in the form of structured data that are not organized in relational database models, such as tables. [8].

- Unstructured data, such as text messages, multimedia, location information, videos, and social media data are data that do not follow a specific format.

The need to analyze and to manage these types of data has become a challenge, due to the size of this type of data. The number of the users of Smartphone, continues to increase and causing large amount of unstructured data. Relational databases from physical infrastructure has found difficult on performance and scalability [8]. Managing system downtime, choosing suitable cloud instances, and choosing a cloud provider are resolved challenges if migrating large database from physical infrastructure into the Google Cloud [10].

Google Cloud has the opportunity to offer to the companies the right amount of services. For example, an organization may want to upgrade the existing database hardware to speed up migration, or schedule downtime to migrate the database while it is idle [10]. Google offers database storage like: Cloud Data store for NoSQL non-relational database, and Cloud SQL for MySQL fully relational database. To choose a database it is important to think about what your data looks like, how you'll query your data, and the scalability you'll need in the future. So, the database will depend on the type and nature of the data. If the data is big in massive volume, they need a performant management. One of the cases that can demonstrate us the best example of Big data is the ecommerce platform.

Today, the e-commerce has become the platform for media and new, unique services and capabilities that aren't found in the physical world [14]. E-commerce is a typical industry most used nowadays being influenced inevitably by the features of cloud computing. It offers services and the possibility to exchange products via the Internet. Ecommerce has two layers: one layer is the technical architecture made up of hardware and software, the other layer is the business transactions based on the technical architecture. Since the data type in this online platform are different like multimedia, image, integer, string etc., it's necessary to have an environment database that can afford storing the flow of all this big data in one place. So, it is important to have a high-level database to manage them. Based on studies of scholarly/academic sources and implementation, Google cloud is going to make a significant impact on e-commerce technical architecture. To store all these data Google Cloud needs to be combine with a database storage based on type of business data. In the next session we will demonstrate how Google cloud has effect on hosting the e-commerce platform. We have compared two databases MongoDB with MySQL to find the best solution for ecommerce platform hosting on Google Cloud.

\section{USE STUDIES- GOOGLE CLOUD SOLUTION FOR E-COMMERCE PLATFORM}

Based on our latest experience with e-commerce platform we found out that we had a problem with performance of data management. Our platform has 270 physical store and operate online to sell the products to the users. The number of users per minute it is too big and the products are sold very fast. One of the most important things is the synchronization of the platform with products stock data every morning. Being that data records for stock file are too large, we had difficult to manage all these records in real time, without losing time and cost. For each store we have to get the stock file from a web service. This file has the product data stock that has to be synchronized to the platform.

At first, we have created MySQL database on cloud to import the stock file and insert the data to the database, but we found it difficult due to the big data on file. For this reason, using MySQL database on cloud can cause lower performance and stability. Based on other implementations and study of scholarly/academic sources we concluded that NoSQL database would be a better solution. We chose to use at the cloud the MongoDB database. One of the most popular NoSQL databases offers multi-document ACID transactions [11]. MongoDB is a database that can be used both in small projects that have several thousands of users, but primarily for products and applications that contain hundreds of thousands of users as our platform is [11].

This e-commerce platform is integrated on Google cloud environment, for security, performance, cost, and scalability. In order to demonstrate the practical use of these types of databases on cloud, we have built two machines on cloud with the same condition, MySQL database and MongoDB database. The aim is to show the performance and scalability between MySQL and MongoDB on Google cloud for ecommerce platform.

Figure 2 and figure 3 shows MySQL and Mongo database on cloud platform, respectively. 


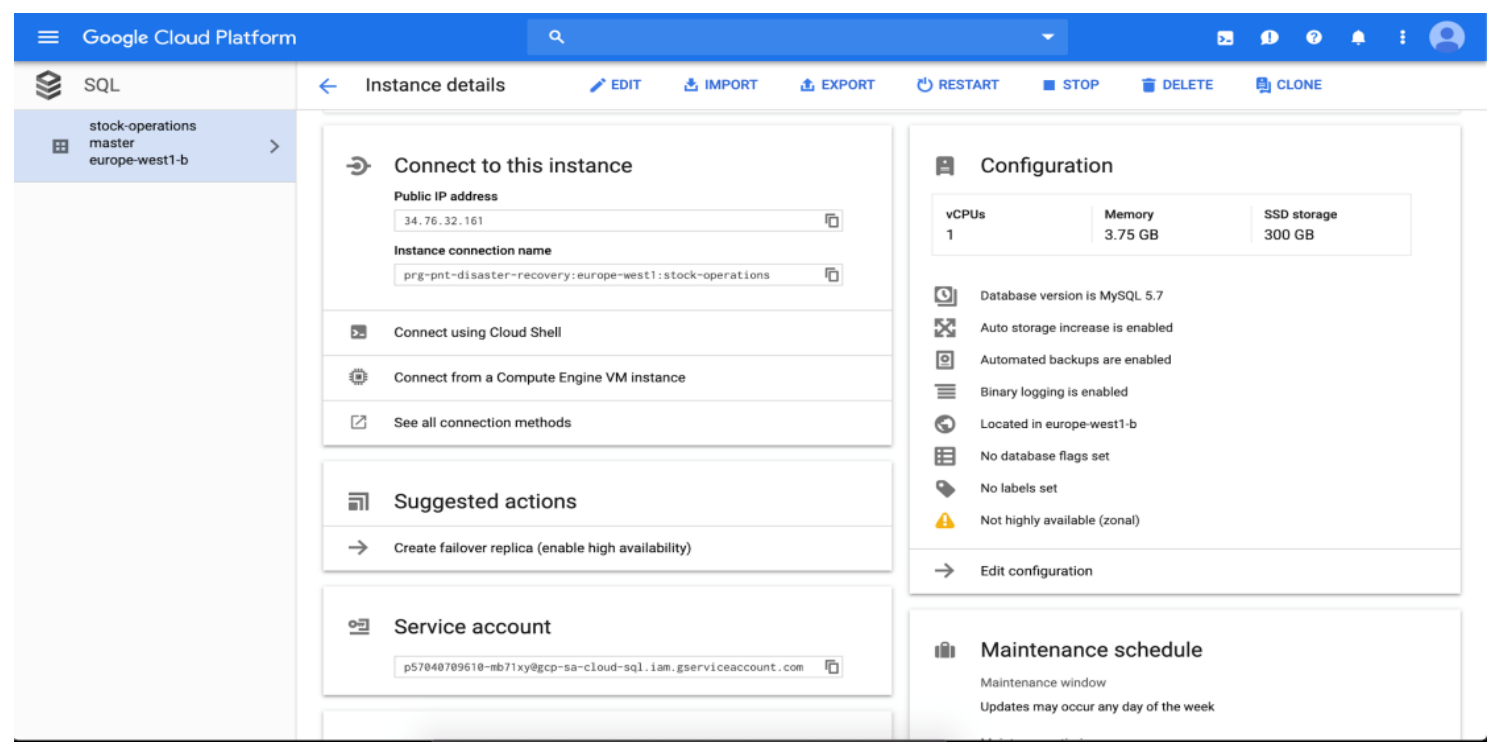

Fig 2. MySQL on Cloud

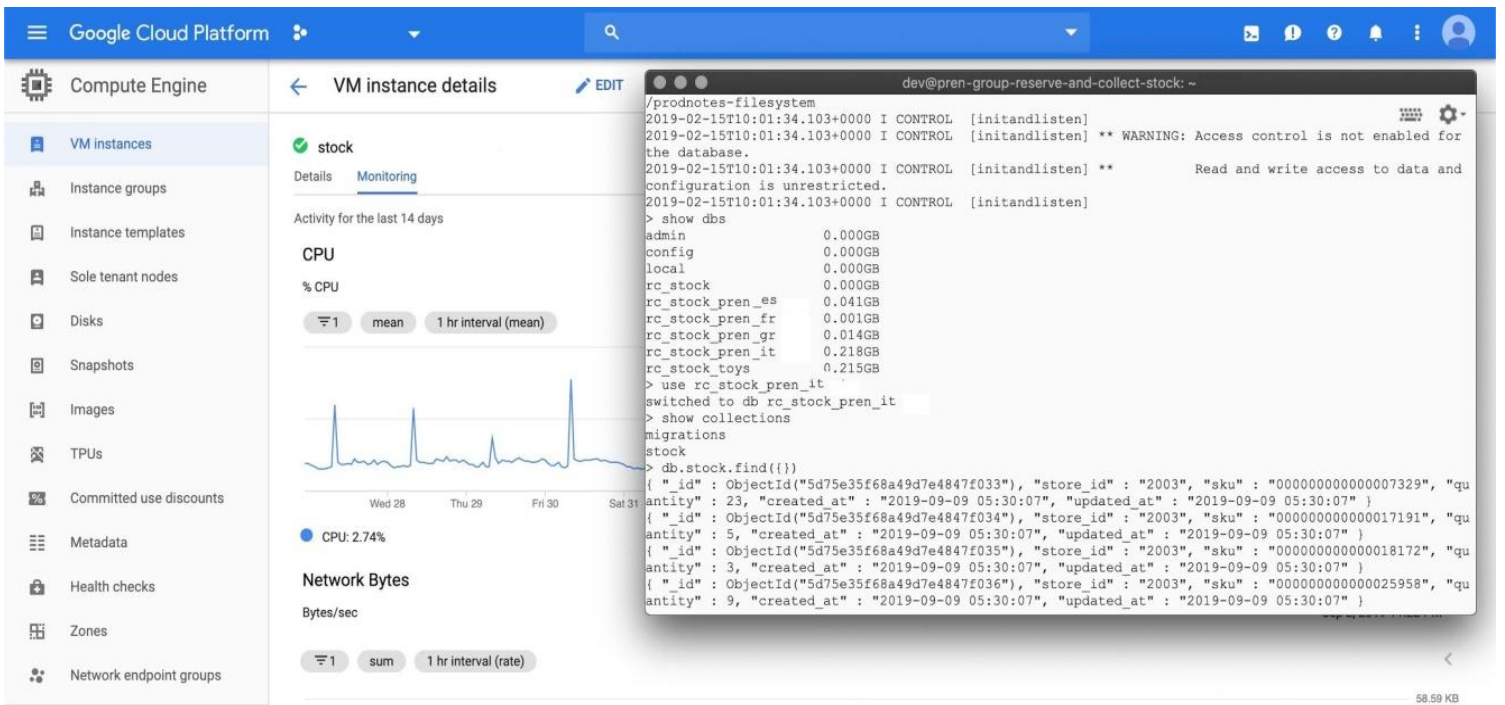

Fig 3. MongoDB on Cloud

The file that we have used is the stock file that has 1440173 records of data. The file columns are: store_id, sku_product,quantity_stock $(2003 ; 000000000000007329 ; 24)$.

We have to import this file into our databases to populate the stock table. After the import of the file we have executed different query both in MySQL and MongoDB to show how these databases will react with this data.

The characteristics for MySQL database are: Machine Type: n1-standard-1 (1 vCPU, 3.75 GB memory),

The characteristics for MongoDB are the same as MySQL: Machine Type: n1-standard-1 (1 vCPU, 3.75 GB memory)

We have created query for the same data to show which is more effective and how is the difference according to the performance.

MySQL database query:

Select $*$

From rc_stock

limit 50000;
To find exactly the response time, we did some queries and, in the table 1 we have put the average response time.

Table 1. Response time in MySQL

\begin{tabular}{|c|c|c|c|}
\hline \multirow{2}{*}{$\begin{array}{c}\text { MySQL } \\
\text { Response }\end{array}$} & \multicolumn{3}{|c|}{ Response time } \\
\cline { 2 - 4 } & Query & Response & Duration \\
\hline Select \\
query & $\begin{array}{c}\text { Select* } \\
\text { FROM rc_stock } \\
\text { limit 50000 }\end{array}$ & $\begin{array}{c}50000 \\
\text { row(s) } \\
\text { returned }\end{array}$ & $0.0547 \mathrm{sec}$ \\
\hline
\end{tabular}

We did the same on MongoDB machine.

Mongo database query:

$>$ show collections

Stock

$>$ db.stock.find().limit(1)

\{ "_id" : ObjectId("5d60cb5f68a49d5ec8019833"), "store_id" : "2133", "sku" : "000000000007537003", "quantity" : 1, 
"created_at" : "2019-08-24 04:30:07", "updated_at" : "201908-24 04:30:07" \}

$>$ db.stock.find().limit(50000)

Table 2 shows the response time for queries executed in MongoDB.

Table 2. Response time in MongoDB

\begin{tabular}{|c|c|c|c|}
\hline \multirow{2}{*}{$\begin{array}{c}\text { MongoRe } \\
\text { sponse }\end{array}$} & \multicolumn{3}{|c|}{ Response time } \\
\cline { 2 - 4 } & Query & Response & Duration \\
\hline $\begin{array}{c}\text { Select } \\
\text { query }\end{array}$ & $\begin{array}{c}\text { db.stock.find() } \\
\text {.limit(50000) }\end{array}$ & "limitAmount" & "executionTime \\
& & & Millis" : 0.067 \\
\hline
\end{tabular}

The response of MongoDB is in millisecond which converted is 0.0134 second.

The difference between MySQL and MongoDB is 0.0412 seconds. This result is shown in the following chart, figure 2 that present for the "select query" results for MySQL and MongoDB.

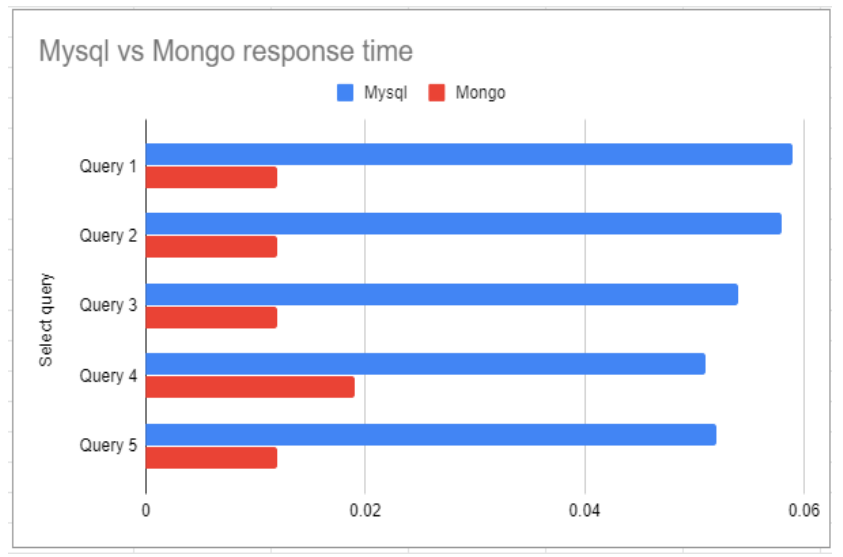

Fig 4. The performance chart for MySQL and MongoDB on cloud

In conclusion, we concluded that using MongoDB for our ecommerce platform was more performant and scalability than MySQL.

MongoDB is available as a fully managed service. Modeling of data in MongoDB database depends on the data and characteristics of MongoDB. Due to variation in data models MongoDB based application performance gets affected. For ecommerce platform is flexible and scalable document database.

\section{CONCLUSIONS}

Google cloud is a platform that offers services for compute, storage, management of big data, security and developer tools. The uses of Cloud enhance performance (efficiency and effectiveness). Cloud has to migrate one database storage for storing data. The size of data at present is huge and continues to increase every day. On cloud we can migrate SQL database for structure data, NoSQL database for unstructured data. Both SQL and NoSQL have their pros and cons. Users must first consider various parameters like query, the interface, availability, redundancy, consistency and analyzing the pros and cons of various data models before choosing a particular data model. It is important to see the type of data and nature of your business. We reviewed the main functionality and security features of two of the most popular SQL and NoSQL databases: MySQL and MongoDB. The main problem for MySQL based on our demonstrated was the performance and scalability due to large and unstructured data on the csv file for our store. After demonstrated we concluded that MongoDB perform better. For large and unstructured data NoSQL databases are often highly optimized for retrieve and append operations and often offer performance beyond record storage.

For the future, Cloud will be most using and popular provider. All the information and data will be on cloud very secure. And we will see NoSQL complement existing applications and take care of the complexity of data management.

\section{REFERENCES}

[1] S. Marston, S. Bandyopadhyay, J.Zhang, A.Ghalsasi, Cloud computing- The business perspective. Decision Support Systems 51(1):176-189. (2011)

[2] I. Ahmad, H. Bakht, U. Mohan: Cloud Computing - A Comprehensive Definition (2017)

[3] L. M. Vaquero, L. Rodero-Merino, J. Caceres, and M Lindner, "A break in the clouds: towards a cloud definition," ACM SIGCOMM Comput. Commun. Rev., vol. 39, no. 1, pp. 50-55, (2008).

[4] Wikipedia- Cloud Computing. [Online]. http://en.wikipedia.org/wiki/Cloud_computing

[5] W,T.Tsai, X.Sun, J.Balasooriya, Service-Oriented Cloud Computing Architecture (2012)

[6] J.Wiley Son ,B. Franks, Taming the Big Data Tidal Wave, Finding Opportunities in Huge Data Streams with Advanced Analytics, (2012).

[7] R.Calinescu, R,F.Paige, Evaluating cloud database migration options using workload models (2018).

[8] I.Abaker, T. Hashem, I.Yaqoob, N, B.Anuar, S.Mokhtar, A.Gani, S,U.Khan, The rise of "big data" on cloud computing: Review and open research issues (2015)

[9] Zhi.Li, S.Bandyopadhyay, J.Zhang, A.Ghalsasi, Cloud computing - The business perspective t $^{2}$ Sean Marston (2011)

[10] Pokorny, J.,:NoSQL databases: a step to database scalability in web environment. 15, 4-15 (2011).

[11] MongoDB. Mongodb. [Online]. Available: http://www.mongodb.org/

[12] Wikipedia- Dedicated hosting (Online)- Dedicated hostingOnlinehttps://en.wikipedia.org/wiki/Dedicated_ho sting_service

[13] Almeida, F.L.F., Calistru, C.: The main challenges and issues of big data management. International Journal of Research Studies in Computing 2, (2012)

[14] A.Vegesna, P.Jain, Dh.Porwal: Ontology based Chatbot (For E-commerce Website), 2018

[15] S.Greca, A. Kosta: Optimizing data retrieval by using MongoDb with Elasticsearch, RTA_CSIT, 2018 